Research Article

\title{
Sentinel Lymph Node Biopsy Is Feasible in Cervical Cancer Laparoscopic Surgery: A Single-Center Retrospective Cohort Study
}

\author{
Hongyi Hou $\left(\mathbb{D}\right.$, Yibo Dai $\mathbb{D}^{D}$, Sichen Liang $(\mathbb{D}$, Zhiqi Wang $(\mathbb{D}$, and Jianliu Wang $(\mathbb{D}$ \\ Department of Obstetrics and Gynecology, Peking University People's Hospital, Beijing, China \\ Correspondence should be addressed to Zhiqi Wang; wangzqnet@sina.com and Jianliu Wang; wangjianliu1203@163.com
}

Received 16 January 2021; Revised 2 March 2021; Accepted 31 March 2021; Published 16 April 2021

Academic Editor: Xiangya Ding

Copyright (c) 2021 Hongyi Hou et al. This is an open access article distributed under the Creative Commons Attribution License, which permits unrestricted use, distribution, and reproduction in any medium, provided the original work is properly cited.

\begin{abstract}
Background and Objective. Sentinel lymph node (SLN) biopsy efficiency has been confirmed in various solid tumors. This study aimed to assess SLN biopsy feasibility in clinical application and explore how to improve its detection rates and diagnostic accuracy in cervical cancer laparoscopic surgery. Methods. A total of 100 cervical cancer patients undergoing laparoscopic surgery with SLN biopsy were included. Indocyanine green, carbon nanoparticles (CNPs), and a combination of both were used during surgeries. Detection rates, sensitivity, negative predictive value (NPV) of SLN biopsy, and related factors were analyzed. Results. The overall and bilateral SLN detection rates were 92\% (92/100) and 74\% (74/100), respectively. Combined tracers had higher bilateral SLN detection rates than CNPs alone $(p=0.005)$. Menopause and lymph node metastasis were associated with lower overall and bilateral SLN detection rates $(p<0.05)$. SLN biopsy sensitivity and NPV for lymph node metastasis in patients with at least one detected SLN were 81.8\% (9/11) and 97.3\% (72/74), respectively. Among those with bilateral detected SLNs, higher sensitivity and NPV of $87.5 \%$ (7/8) and 98.3\% (57/58) were observed, respectively. SLN algorithm can ensure that all patients with lymph node metastasis are detected by SLN biopsy. Conclusion. SLN biopsy appears to be safe and effective for specific cervical cancer patients with high detection rates and NPV in laparoscopic surgery, especially for those with detected bilateral SLNs and undergoing the SLN algorithm. Selecting suitable patients for SLN mapping has prospects for clinical application.
\end{abstract}

\section{Introduction}

Cervical cancer is one of the most common gynecologic malignancies around the world $[1,2]$. Since lymph node metastasis is one of the most important prognostic predictors of early-stage cervical cancer, it is closely associated with adjuvant therapy strategy $[3,4]$, thus making lymph node resection the most important step in comprehensive surgical staging of cervical cancer. Nevertheless, lymph node positivity rate is relatively low in early-stage cervical cancer patients. In addition, systemic lymph node resection is associated with longer surgical time, higher bleeding volume, higher rates of intraoperative subsidiary injures, and destruction of normal barrier functions in the lymph nodes [5].

SLN biopsy has gained popularity in recent years. Since first introduced in the 1960s, studies on SLN have been performed in breast cancer, melanoma, and vulvar cancer, and standards for SLN biopsy have been set up in clinical practice. SLN biopsy is now recommended by the National Comprehensive Cancer Network (NCCN) guidelines for stage I cervical cancer patients (category 2A) [6]. However, there is still no consensus on the standard SLN biopsy method in cervical cancer, and differences exist among current study results.

Based on retrospective data from Peking University People's Hospital, the present study compared different tracers and analyzed clinicopathological factors associated with SLN detection rates, sensitivity, and NPV for SLN biopsy in cervical cancer laparoscopic surgery.

\section{Materials and Methods}

2.1. Patient Selection and Data Collection. One hundred pathologically confirmed cervical cancer patients who 
underwent SLN mapping during laparoscopic surgeries in Peking University People's Hospital from July 2015 to June 2018 were retrospectively reviewed. Clinicopathological information was collected, including age, body mass index, gravidity, parity, medical history, history of cervical conization, neoadjuvant chemotherapy, preoperative radiotherapy, radiography, surgeries, SLN tracers, numbers of resected lymph nodes, stage, tumor size, histology, grade, lymphovascular space invasion, depth of cervical stromal invasion, and lymph node metastasis. The study was approved by the Institutional Review Board of Peking University People's Hospital (approval number: 2018PHD003-01).

2.2. Reagents and Instruments. CNPs injection $(50 \mathrm{mg} / 1 \mathrm{ml}$ per piece) was from Chongqing Lummy Pharmaceutical Co., Ltd. (approval number: H20041829). Indocyanine green (ICG) injection (25 mg per piece) was from (Liaoning) Pharmaceutical Co., Ltd. and was dissolved in $10 \mathrm{ml} 0.9 \% \mathrm{NaCl}$ solution before use. The intraoperative fluorescence imaging system (PC9000) was from Novadaq Technologies Inc.

2.3. Surgical Procedures. All 100 eligible patients received laparoscopic surgery. After successful anesthesia, SLN tracers were injected into the cervix. In the single-tracer method, $0.2 \mathrm{ml} \mathrm{CNPs}$ or ICG solution was injected into 3, 6 , 9 , and 12 o'clock of the cervix with a depth of $2-3 \mathrm{~mm}$, respectively $(0.8 \mathrm{ml}$ totally). In the combination-tracing method, CNPs were injected into 3, 6, 9, and 12 o'clock firstly, followed by the injection of ICG solution to 2, 4, 8, and 10 o'clock of the cervix. Tracers were injected near the tumor to avoid being injected into the tumor tissue. After injection, the cervix was compressed for 30 seconds.

During the surgery, the retroperitoneum was dissected, and SLNs were searched along the lymphatic drainage pathway. The anatomical sites, numbers of SLNs, and time to detection were recorded. According to the NCCN guidelines' SLN algorithm [6], all detectable SLNs and enlarged or suspicious nodes regardless of mapping were removed; if no SLN was detected in either side of the pelvis, a side-specific systemic lymph node resection was conducted; the tumor and parametrium were resected completely. The mapped SLNs, lymph vessels, and uterine are shown in Figure 1.

For stage IA1 patients with no lymphovascular space invasion in the preoperative biopsy, extrafascial hysterectomy and SLN biopsy were conducted. For stage IA1 patients with lymphovascular space invasion or higher stages, radical hysterectomy and systemic pelvic lymph node dissection were performed after SLN resection, and para-aortic lymph node biopsy was conducted when necessary. Systemically resected pelvic lymph nodes included external iliac, internal iliac, obturator fossa, deep inguinal, and common iliac lymph nodes.

All surgeries were performed by three experienced gynecologic oncologists in our center.

2.4. Pathological Review. Routine hemotoxin and eosin staining was used. We did not conduct the SLN ultrastaging and immunohistochemistry staining in this study. All pathological reviews were finished by two independent gynecologic pathologists in the Department of Pathology of Peking University People's Hospital. When discordance occurred, a third senior pathologist was invited to decide the diagnosis.

2.5. Evaluation of SLN Biopsy. Overall SLN detection rate refers to the percentage of patients with successfully detected SLNs in all eligible patients. Bilateral SLN detection rate refers to the percentage of patients with successfully detected SLNs on both sides of pelvis in all eligible patients. Sensitivity refers to the percentage of patients with positive SLNs in routine pathological examination among those with positive pelvic lymph nodes. NPV refers to the percentage of patients with negative pelvic lymph nodes among those whose SLNs were negative in routine pathological examination.

2.6. Statistical Methods. The $\chi^{2}$ test (with continuity adjustment) and Fisher's exact test were used for comparing categorical variables, and partition of $\chi^{2}$ test (Bonferroni method) was used for intergroup comparisons. Student's $t$ test was used for comparing continuous variables. Multivariable logistic regression was conducted to analyze independent predictors of SLN detection rates. All statistical analyses were performed using Statistical Package for the Social Sciences (SPSS) Software 23.0 (IBM Corporation, Armonk, NY). All $p$ values were two-sided, and $p<0.05$ was considered statistically significant.

\section{Results}

The clinicopathologic characteristics of 100 eligible patients are listed in Table 1. There were no previous histories of malignant tumors, pelvic lymphadenectomy, inguinal lymphadenectomy, or other surgeries that might change uterine lymphatic drainage in the 100 patients. The staging of all cases was based on the 2018 International Federation of Gynecology and Obstetrics (FIGO) staging system [7]. 2,875 pelvic lymph nodes were removed during surgeries, and the median number of pelvic lymph nodes removed was 29 (range: 24-35) in each patient. The median surgical time to complete the SLN mapping was $30 \mathrm{~min}$ (range: $25-40 \mathrm{~min}$ ). A total of 722 sentinel lymph nodes were removed, with a median number of 7 (range: $4-11$ ) for each patient. The most common location of SLNs was the external iliac region (277/ $722,38.4 \%)$, followed by obturator fossa $(256 / 722,35.5 \%)$, common iliac $(71 / 722,9.8 \%)$, internal iliac $(71 / 722,9.8 \%)$, deep inguinal $(22 / 722,3.0 \%)$, presacral $(9 / 722,1.2 \%)$, inferior vena cava $(8 / 722,1.1 \%)$, para-aortic $(4 / 722,0.6 \%)$, and parametrial $(4 / 722,0.6 \%)$ regions. The distribution of SLNs is presented in Figure 2.

The overall detection rate of SLNs was 92\% (92/100). SLNs were found on both sides of the pelvis in 74 patients (74\%). There were 85 patients undergoing combined tracers of ICG and CNPs, 8 patients with ICG tracer alone, and 7 patients undergoing CNPs tracer alone. The SLN detection 


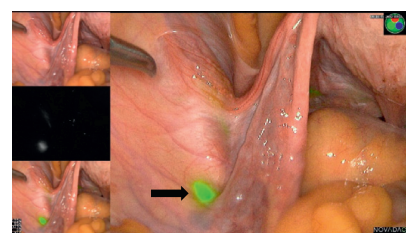

(a)

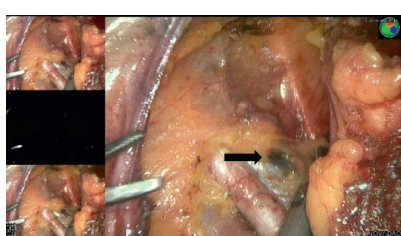

(b)

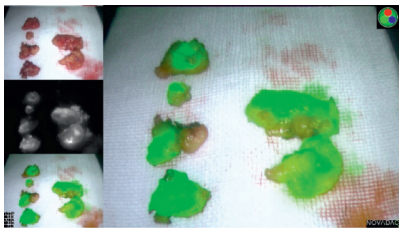

(e)

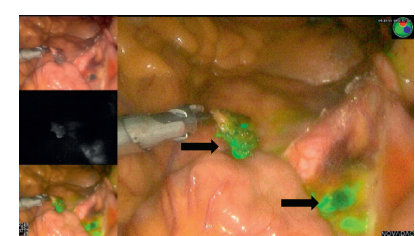

(c)

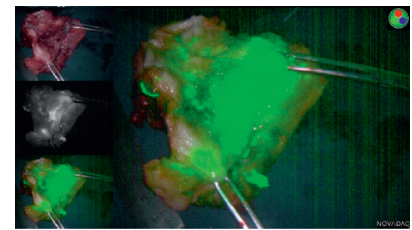

(f)

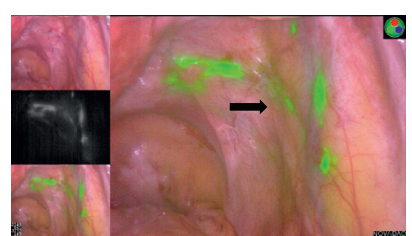

(d)

Figure 1: The mapped sentinel lymph nodes, lymph vessels, and uterus. (a) The sentinel lymph nodes mapped by ICG. (b) The sentinel lymph nodes mapped by CNPs. (c) The sentinel lymph nodes mapped by ICG and CNPs. (d) The lymph vessels mapped by ICG. (e) The resected sentinel lymph nodes detected by ICG. (f) The removed uterus injected by ICG.

rates according to the different tracer methods are listed in Table 2. The percentage of patients with at least one detected SLN did not differ significantly among the three groups $(p=0.262)$. However, the difference of bilateral detection rates among the three groups was statistically significant $(p=0.014)$. Bonferroni-corrected threshold $\alpha=0.0167$ was applied in analyses stratified according to different tracers. The bilateral detection rate was significantly higher by combined tracers compared with CNPs tracer alone (75.3\% versus $54.3 \%, p=0.005$ ).

Clinicopathological factors influencing the detection rates of SLNs were analyzed (Table S1). Factors with $p<0.1$ in univariate analyses or those with potential influence according to our clinical experience were included in the logistic regression model. The results suggested that menopause $(\mathrm{OR}=0.02,95 \% \mathrm{CI} 0.001-0.47, p=0.016 ; \mathrm{OR}=0.30$, $95 \%$ CI $0.09-0.93, p=0.038$ ) and lymph node metastasis $(\mathrm{OR}=0.03,95 \%$ CI $0.002-0.71, p=0.030 ; \mathrm{OR}=0.17,95 \% \mathrm{CI}$ $0.03-0.92, p=0.040)$ were associated with lower overall and bilateral SLN detection rates (Table 3). The overall SLN detection rate was $98.4 \%$ in premenopausal patients and $82.1 \%$ in postmenopausal patients, and the bilateral SLN detection rates were $82.0 \%$ and $61.5 \%$, respectively. The patients with positive pelvic lymph nodes had lower overall and bilateral SLN detection rates compared with those without positive lymph nodes (73.3\% versus $95.3 \%$; 53.3\% versus $77.6 \%$ ).

The results of SLN biopsy and pelvic lymphadenectomy are shown in Figure 3. Among the 83 patients with at least one SLN detected undergoing SLN mapping followed by systemic pelvic lymphadenectomy, 9 had positive SLNs, and 2 cases were false negative. Therefore, the sensitivity and NPV of SLN biopsy were $81.8 \%(9 / 11)$ and $97.3 \%$ (72/74), respectively. Among the 65 patients with bilateral SLNs detected, one had a false-negative result, yielding a sensitivity of $87.5 \%$ (7/8) and an NPV of $98.3 \%$ (57/58). Diagnostic accuracy of SLN biopsy is listed in Table 4.

Two patients showed false-negative results. A total of 8 negative SLNs were detected in patient A, located in the left external iliac, right external iliac, right internal iliac, and right obturator region. But a single positive non-SLN was found in right parametrial area. Patient $\mathrm{B}$ had 3 negative SLNs detected in the right external iliac and right para-aortic region, but 5 positive non-SLNs were found in the left hemipelvis (left obturator region). If the NCCN guidelines' SLN algorithm had been applied in this cohort, the false-negative patient seems to be 0 and the sensitivity and NPV are $100 \%$.

SLNs were detected in 148 hemi-pelvises. 13 hemipelvises had positive lymph nodes, 10 of which had positive SLNs. Side-specific sensitivity was $76.9 \%(10 / 13)$, and NPV was $97.8 \%$ (135/138). The 3 false-negative hemipelvises were from 3 different patients. Patient $\mathrm{C}$ had one positive non-SLN located in the right obturator region, 4 negative SLNs located in the right common iliac and right external iliac area, and 3 positive SLNs located in the left common iliac and left obturator region. There was one positive non-SLN in the right obturator region of patient $\mathrm{D}$ who had 2 negative SLNs in the right obturator and left internal iliac region and 2 positive SLNs in the left internal iliac region. The third patient was the above patient A. Lymph node statuses of the four patients are shown in Figure 4.

\section{Discussion}

This study aimed to explore the detection rates, diagnostic accuracy, and the associated factors of SLN biopsy in order to analyze its feasibility and clinical value in cervical cancer. The overall and bilateral SLN detection rates of $92 \%$ and $75 \%$ were observed, which were generally consistent with the results of previous studies [8-10]. Combined technique could bring a higher bilateral detection rate than CNPs alone but not ICG alone. Patients after their menopause or with metastatic pelvic lymph nodes presented the significantly lower overall and bilateral SLN detection rates. When SLNs were detected on bilateral pelvis and SLN mapping algorithm was adhered to, SLN biopsy was more reliable.

Near-infrared fluorescence imaging has the potential for the identification of anatomical structures covered under a layer of fatty tissue, with a maximum penetration depth of 
TABLE 1: Clinicopathological characteristics in 100 cervical cancer patients.

\begin{tabular}{|c|c|}
\hline Characteristic & Value \\
\hline Mean age (years) & $47.2 \pm 9.7$ \\
\hline \multicolumn{2}{|l|}{ Menopause } \\
\hline No & $61(61.0)$ \\
\hline Yes & $39(39.0)$ \\
\hline Mean body mass index $\left(\mathrm{kg} / \mathrm{m}^{2}\right)$ & $23.9 \pm 3.3$ \\
\hline Median gravidity & $3(2.4)$ \\
\hline Median parity & $1(1.2)$ \\
\hline \multicolumn{2}{|l|}{ Conization history } \\
\hline No & $85(85.0)$ \\
\hline Yes & $15(15.0)$ \\
\hline \multicolumn{2}{|l|}{ Neoadjuvant chemotherapy } \\
\hline No & $76(76.0)$ \\
\hline Yes & $24(24.0)$ \\
\hline \multicolumn{2}{|l|}{ Preoperative radiotherapy } \\
\hline No & $97(97.0)$ \\
\hline Yes & $3(3.0)$ \\
\hline \multicolumn{2}{|l|}{ Sentinel lymph node biopsy only } \\
\hline No & $91(91.0)$ \\
\hline Yes & $9(9.0)$ \\
\hline \multicolumn{2}{|l|}{ Stage } \\
\hline IA1 & $11(11.0)$ \\
\hline IA2 & $3(3.0)$ \\
\hline IB1 & $27(27.0)$ \\
\hline IB2 & $26(26.0)$ \\
\hline IB3 & $9(9.0)$ \\
\hline IIA1 & $5(5.0)$ \\
\hline IIA2 & $3(3.0)$ \\
\hline IIB & $1(1.0)$ \\
\hline IIIC1 & $13(13.0)$ \\
\hline IIIC2 & $2(2.0)$ \\
\hline \multicolumn{2}{|l|}{ Tumor size $(\mathrm{cm})$} \\
\hline$<2$ & $45(45.0)$ \\
\hline$\geq 2 \sim<4$ & $39(39.0)$ \\
\hline$\geq 4$ & $16(16.0)$ \\
\hline \multicolumn{2}{|l|}{ Histologic type } \\
\hline Squamous carcinoma & $76(76.0)$ \\
\hline Adenocarcinoma & $14(14.0)$ \\
\hline Adenosquamous carcinoma & $4(4.0)$ \\
\hline Others & $6(6.0)$ \\
\hline \multicolumn{2}{|l|}{ Grade } \\
\hline 1 & $26(26.0)$ \\
\hline 2 & $41(41.0)$ \\
\hline 3 & $33(33.0)$ \\
\hline \multicolumn{2}{|l|}{ Lymphovascular space invasion } \\
\hline No & $52(52.0)$ \\
\hline Yes & $48(48.0)$ \\
\hline \multicolumn{2}{|l|}{ Depth of cervical invasion } \\
\hline$<1 / 2$ & $62(62.0)$ \\
\hline$\geq 1 / 2$ & $38(38.0)$ \\
\hline \multicolumn{2}{|l|}{ Lymph node status } \\
\hline Negative & $85(85.0)$ \\
\hline Positive & $15(15.0)$ \\
\hline
\end{tabular}

The values were presented as mean \pm standard deviation, median (minimum, maximum), or number (\%), unless otherwise indicated.

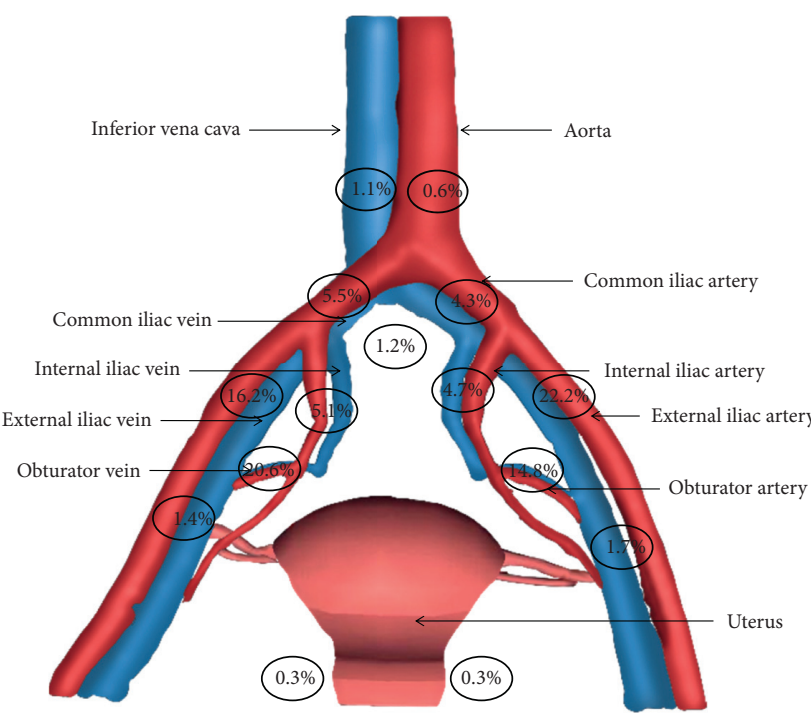

Figure 2: The distribution of sentinel lymph nodes.

$10 \mathrm{~mm}$ [11]. Previous studies emphasized the advantages of ICG over other tracers for better SLN detection in cervical and endometrial cancer, with the reported detection rates ranging from $78 \%$ to $86 \%$ [12-14]. Imboden et al. [15] used Tc-99 radiotracer combined with blue dye for SLN mapping in a group of 36 cervical cancer patients and ICG alone in another group of 22 patients. The overall detection rate for dual injection was $83 \%$, while for ICG alone it was $95.5 \%$. The bilateral detection rate was $61 \%$ and $95.5 \%$, respectively $(p<0.05)$. In a meta-analysis of 6 studies, including 538 patients with cervical and endometrial cancers, the overall and bilateral SLN detection rates were both significantly higher in the ICG group than those in the blue dye group $(p<0.001)$. Meanwhile the advantage was not shown when compared with Tc-99 alone or combined use of blue dye and Tc-99 $(p>0.05)[16]$.

We found that menopausal state was associated with lower SLN detection rates, which was, to some extent, in accordance with previous findings [8, 17]. Possible reasons include weakened lymphatic drainage in atrophy of the cervix with the decline of estrogen level after menopause, which might affect the tracers' spread. Lymph node metastasis was also proved to influence the SLN detection by previous studies [18]. In the study by Malur et al. [19], the SLNs in 50 patients with cervical cancer were mapped with Tc-99 and/or blue dye. The overall SLN detection rate was $78 \%$ (39/50). Among the 10 patients with metastatic lymph nodes, 4 patients had no SLNs detected. Tumor thrombus will obstruct and redistribute lymphatic drainage so that tracers fail to reach SLNs normally along the lymphatic vessels, leading to the failure of SLN mapping. In breast cancer and penile cancer, this hypothesis was generally accepted, and SLN biopsy was not applicable if there were suspected metastatic lymph nodes in preoperative assessment [20-22]. So it is imperative to identify the patients with 
TABLE 2: Sentinel lymph node detection rates according to different tracer methods.

\begin{tabular}{lcccccc}
\hline Tracer & $N$ & Overall detection rate $(n, \%)$ & $\chi^{2}$ & $p$ value & Bilateral detection rate $(n, \%)$ & $\chi^{2}$ \\
\hline ICG & 93 & $82,88.2$ & 2.702 & 0.262 & $61,65.6$ & 8.537 \\
CNPs & 92 & $77,83.7$ & & & $50,54.3^{*}$ & 0.014 \\
ICG + CNPs & 85 & $78,91.8$ & & & $64,75.3^{*}$ & \\
\hline
\end{tabular}

The 93 cases of ICG included 85 with combined tracers and 8 with ICG alone. The 92 cases of CNPs included 85 with combined tracers and 7 with CNPs alone. ICG detected at least one SLN in 82 patients including 75 with combined tracers and 7 with ICG alone. ICG detected bilateral SLNs in 61 patients including 55 with combined tracers and 6 with ICG alone. CNPs detected at least one SLN in 77 patients including 70 with combined tracers and 7 with CNPs alone. CNPs detected bilateral SLNs in 50 patients including 46 with combined tracers and 4 with CNPs alone. ${ }^{*}$ CNPs versus ICG + CNPs, $p=0.005$. SLN, sentinel lymph node; ICG, indocyanine green; CNPs, carbon nanoparticles.

TABLE 3: Logistic regression for factors affecting overall and bilateral detection rates of sentinel lymph nodes.

\begin{tabular}{|c|c|c|c|c|}
\hline \multirow{2}{*}{ Characteristic } & \multicolumn{2}{|c|}{ Overall detection rate } & \multicolumn{2}{|c|}{ Bilateral detection rate } \\
\hline & OR $(95 \% \mathrm{CI})$ & $p$ value & OR $(95 \% \mathrm{CI})$ & $p$ value \\
\hline \multicolumn{5}{|l|}{ Menopause } \\
\hline No & 1 & & 1 & \\
\hline Yes & $0.02(0.001-0.47)$ & 0.016 & $0.30(0.09-0.93)$ & 0.038 \\
\hline \multicolumn{5}{|c|}{ Body mass index $\left(\mathrm{kg} / \mathrm{m}^{2}\right)$} \\
\hline$<25.0$ & 1 & & 1 & \\
\hline$\geq 25.0$ & $1.00(0.13-7.84)$ & 0.997 & $2.07(0.66-6.45)$ & 0.212 \\
\hline \multicolumn{5}{|l|}{ Conization history } \\
\hline No & 1 & & 1 & \\
\hline Yes & & 0.999 & & 0.998 \\
\hline Tumor size $(\mathrm{cm})$ & & 0.186 & & 0.221 \\
\hline$<2$ & 1 & & 1 & \\
\hline$\geq 2 \sim<4$ & $0.04(0.001-1.28)$ & 0.068 & $1.20(0.28-5.13)$ & 0.803 \\
\hline$\geq 4$ & $0.37(0.01-13.14)$ & 0.583 & $0.36(0.08-1.56)$ & 0.172 \\
\hline Grade & & 0.313 & & 0.089 \\
\hline 1 & 1 & & 1 & \\
\hline 2 & & 0.998 & $0.17(0.03-1.06)$ & 0.058 \\
\hline 3 & & 0.998 & $0.49(0.07-3.19)$ & 0.451 \\
\hline \multicolumn{5}{|c|}{ Lymphovascular space invasion } \\
\hline No & 1 & & 1 & \\
\hline Yes & $9.37(0.48-182.95)$ & 0.140 & $3.07(0.74-12.72)$ & 0.121 \\
\hline \multicolumn{5}{|c|}{ Depth of cervical invasion } \\
\hline$<1 / 2$ & 1 & & 1 & \\
\hline$\geq 1 / 2$ & $0.78(0.04-14.51)$ & 0.865 & $0.73(0.18-2.97)$ & 0.662 \\
\hline \multicolumn{5}{|c|}{ Lymph node metastasis } \\
\hline No & 1 & & 1 & \\
\hline Yes & $0.03(0.002-0.71)$ & 0.030 & $0.17(0.03-0.92)$ & 0.040 \\
\hline
\end{tabular}

high risk of lymph node metastasis before cervical cancer surgery and assess the feasibility of SLN biopsy.

Diagnostic accuracy of SLN biopsy procedure is critical and should be as high as possible. To reduce the false-negative rate, detecting SLNs on both sides of the pelvis is an essential factor. The study by Lecuru and colleagues supported this view [8]. Among 139 patients of early-stage cervical cancer, 2 cases were false negative, generating a sensitivity of $92 \%$ and an NPV of $98.2 \%$. However, among the 104 patients with SLNs detected bilaterally, none had a false-negative result. In a study conducted by AGO, the sensitivity of SLN biopsy increased from $69.6 \%$ to $87.2 \%$ in patients with bilaterally detected SLNs, compared with those whose SLNs were mapped in one side of the pelvis $(p=0.046)$. An increase in NPV from $91.0 \%$ to $96.5 \%(p=0.062)$ was also observed [23]. Our results further supported the above findings. Besides, in previous studies, SLN ultrastaging and molecular detection methods also increased the diagnostic accuracy of SLN biopsy $[23,24]$. Nevertheless, the effects of micrometastasis and isolated tumor cells of lymph nodes on cervical cancer prognosis were still debatable.

NCCN guidelines' SLN mapping algorithm is fundamental for SLN biopsy to replace systemic lymphadenectomy in patients with early-stage cervical cancer [6]. Cormier's [9] study on SLN mapping algorithm for earlystage cervical cancer showed that, among 122 patients, 3 cases were false negative. Two cases had positive non-SLNs located in the parametrial area, and the positive non-SLNs of another case were at the side without SLNs detected. If the SLN mapping algorithm was implemented, the three false-negative patients could be identified and receive necessary adjuvant treatment. According to the SLN algorithm, the two false-negative patients in our study, patient A and patient $B$, could be identified, so that the sensitivity and NPV 


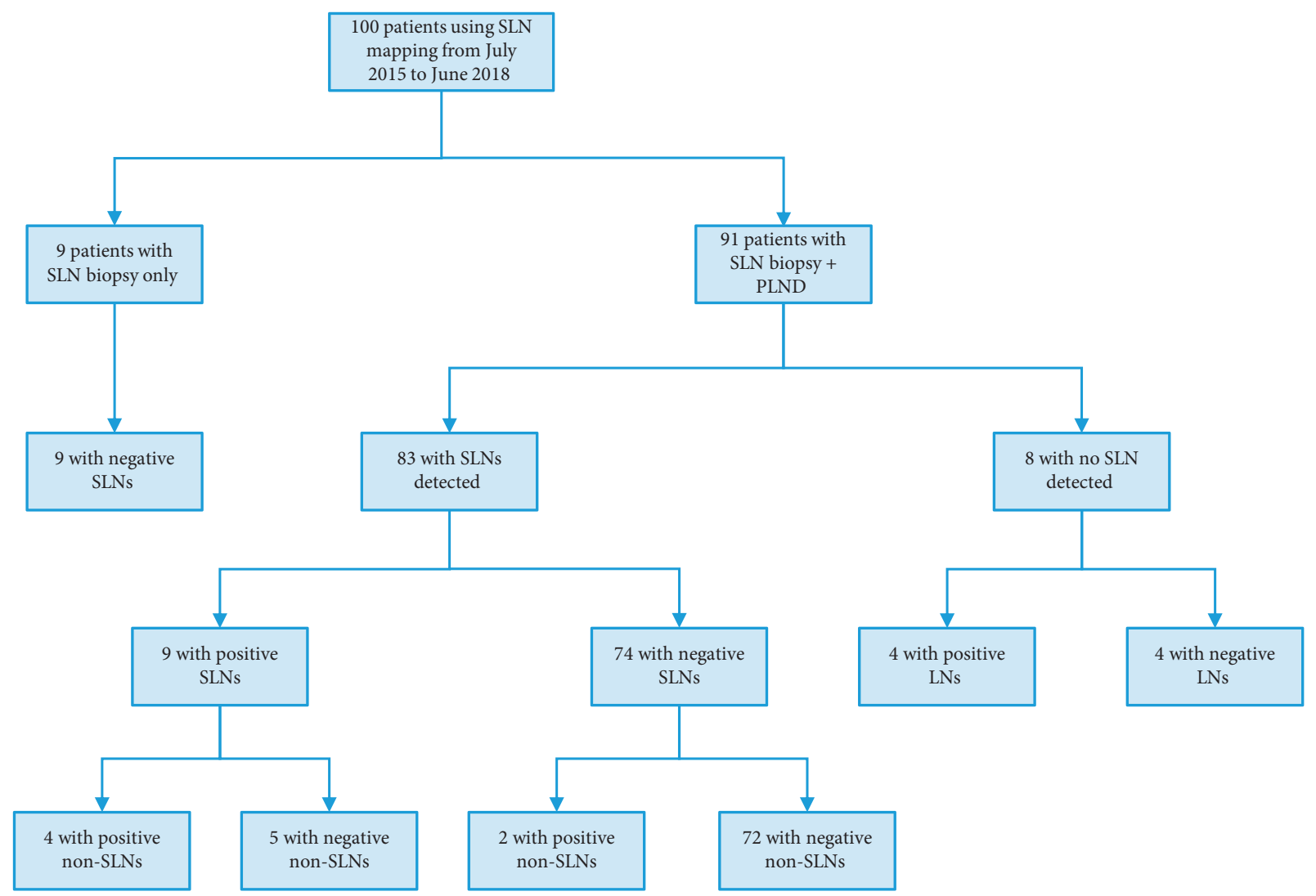

FIGURE 3: The results of SLN biopsy and pelvic lymphadenectomy. SLN, sentinel lymph node; PLND, pelvic lymph node dissection; LN, lymph node.

TABle 4: Diagnostic accuracy of lymph node metastasis using SLN biopsy.

\begin{tabular}{|c|c|c|c|c|c|c|c|c|c|c|c|c|c|}
\hline \multicolumn{8}{|c|}{83 patients with at least one SLN detected } & & \multicolumn{5}{|c|}{65 patients with bilateral SLNs detected } \\
\hline & & \multicolumn{2}{|c|}{$\begin{array}{c}\text { Pelvic } \\
\text { lymph } \\
\text { node }\end{array}$} & \multirow[t]{2}{*}{$n$} & \multirow[t]{2}{*}{ Sensitivity } & \multirow[t]{2}{*}{ NPV } & & & \multicolumn{2}{|c|}{$\begin{array}{c}\text { Pelvic } \\
\text { lymph } \\
\text { node }\end{array}$} & \multirow[t]{2}{*}{$n$} & \multirow[t]{2}{*}{ Sensitivity } & \multirow[t]{2}{*}{ NPV } \\
\hline & & + & - & & & & & & + & - & & & \\
\hline \multirow{2}{*}{ SLN } & + & 9 & 0 & 9 & & & \multirow{2}{*}{ SLN } & + & 7 & 0 & 7 & & \\
\hline & - & 2 & 72 & 74 & $81.8 \%(9 / 11)$ & $97.3 \%(72 / 74)$ & & - & 1 & 57 & 58 & $87.5 \%(7 / 8)$ & $98.3 \%(57 / 58)$ \\
\hline$n$ & & 11 & 72 & 83 & & & $n$ & & 8 & 57 & 65 & & \\
\hline
\end{tabular}

+, positive; -, negative. SLN, sentinel lymph node; NPV, negative predictive value.

were both $100 \%$. As for patients $\mathrm{C}$ and $\mathrm{D}$, the final lymph node status was not affected, even though they had falsenegative hemi-pelvises. Both of them had neoadjuvant chemotherapy history and suspected enlarged pelvic lymph nodes shown by pelvic Magnetic Resonance Imaging before surgery. A previous study thought that neoadjuvant chemotherapy could reduce the detection of pelvic lymph node metastasis [19].

In this study, all surgeries were done by 3 experienced gynecologic oncologists from our center, which guaranteed consistency and comparability among cases. The detailed sites of SLNs were documented for all patients, which helped increase the accuracy of this study; and we found some significant clinicopathological factors associated with SLN detection rates in cervical cancer, which may help screen suitable candidates for SLN biopsy in the future. But there were still some limitations. Firstly, the cohort's size was relatively small, especially as to those treated with one single tracer or patients with specific and advanced clinicopathological features. No significant influence of these factors on SLN detection rates was observed, possibly due to the limited sample size. Secondly, all surgeries were done by the laparoscopic approach in this study. Further studies exploring the application of SLN biopsy in open surgery may be necessary. Thirdly, as a retrospective research, the study is limited in controlling confounders. But statistical methods were adopted, and the results from our work may be helpful for further prospective cohort design. 


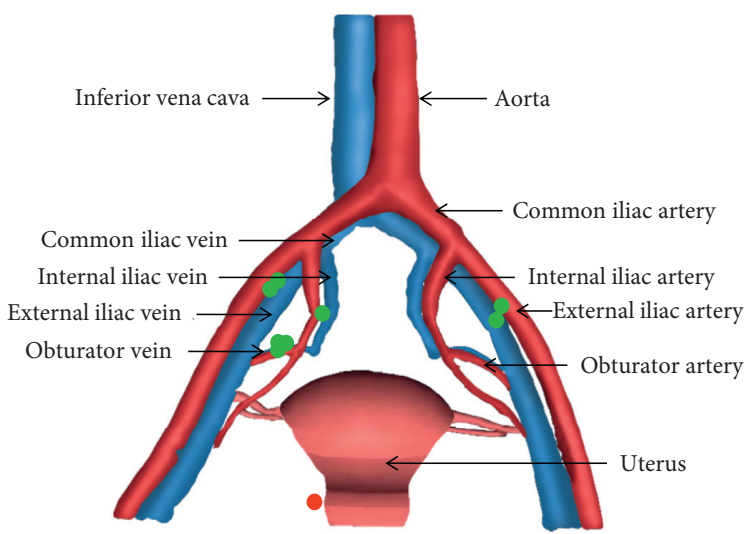

(a)

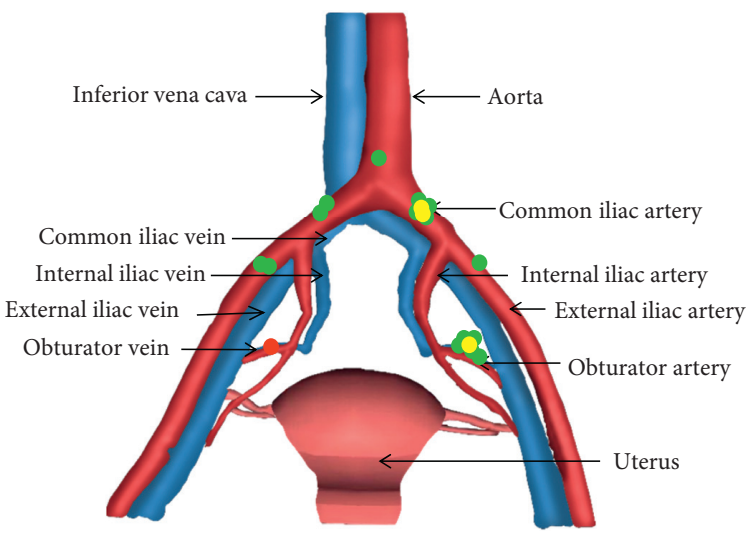

(c)

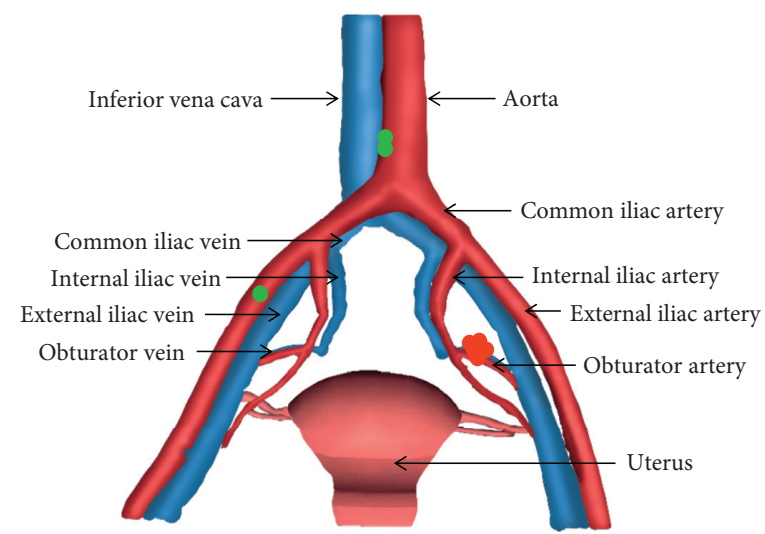

(b)

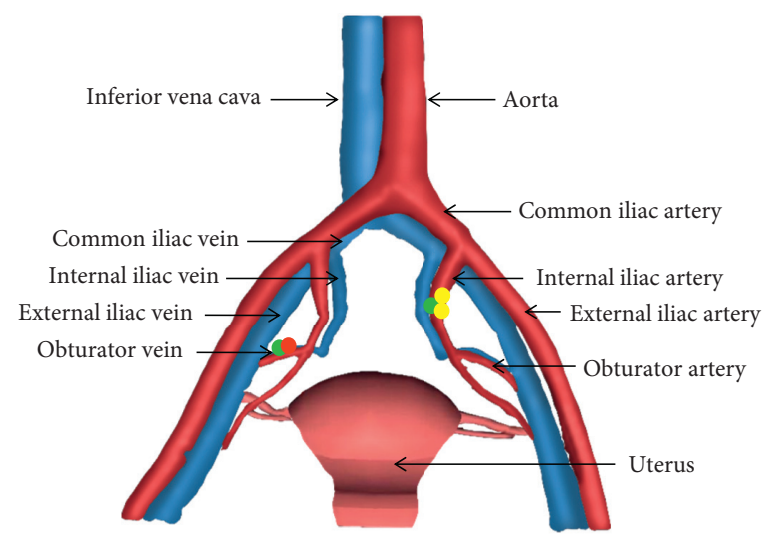

(d)

FIgURE 4: The patients with false-negative sentinel lymph nodes, $\bullet$ positive non-sentinel lymph nodes, $\bullet$ negative sentinel lymph nodes and positive sentinel lymph nodes. (a) Patient A. (b) Patient B. (c) Patient C. (d) Patient D.

\section{Conclusions}

In conclusion, SLN biopsy is feasible in cervical cancer laparoscopic surgery. Premenopausal patients or those without lymph node metastasis have higher SLN detection rates. Detecting SLNs bilaterally and following SLN mapping algorithm make SLN biopsy more reliable. A larger prospective cohort is essential for further demonstrating these results.

\section{Data Availability}

The data are available upon request to the corresponding author.

\section{Ethical Approval}

The study was approved by the Institutional Review Board of Peking University People's Hospital (approval number: 2018PHD003-01).

\section{Conflicts of Interest}

The authors declare that there are no conflicts of interest relevant to this article.

\section{Acknowledgments}

This work was supported by the National Natural Science Foundation of China (81972426) and National Key Technology R\&D Program of China (2019YFC1005200 and 2019YFC1005201).

\section{Supplementary Materials}

The clinicopathological factors influencing the detection rates of SLNs were analyzed in the supplementary file. (Supplementary Materials)

\section{References}

[1] R. L. Siegel, K. D. Miller, and A. Jemal, "Cancer statistics, 2020," CA: A Cancer Journal for Clinicians, vol. 70, no. 1, pp. 7-30, 2020.

[2] J. Ferlay, M. Colombet, I. Soerjomataram et al., "Cancer incidence and mortality patterns in Europe: estimates for 40 countries and 25 major cancers in 2018," European Journal of Cancer, vol. 103, pp. 356-387, 2018.

[3] O. K. Macdonald, J. Chen, M. Dodson, C. M. Lee, and D. K. Gaffney, "Prognostic significance of histology and positive lymph node involvement following radical hysterectomy in carcinoma of the cervix," American Journal of Clinical Oncology, vol. 32, no. 4, pp. 411-416, 2009. 
[4] P. Biewenga, J. van der Velden, B. W. J. Mol et al., "Prognostic model for survival in patients with early stage cervical cancer," Cancer, vol. 117, no. 4, pp. 768-776, 2011.

[5] L. A. Torre, F. Islami, R. L. Siegel, E. M. Ward, and A. Jemal, "Global cancer in women: burden and trends," Cancer Epidemiology Biomarkers \& Prevention, vol. 26, no. 4, pp. 444457, 2017.

[6] National Comprehensive Cancer Network, NCCN Clinical Practice Guidelines in Oncology: Cervical Cancer, National Cancer Institute, Bethesda, MD, USA, http://www.nccn.org.

[7] N. Bhatla, D. Aoki, D. N. Sharma, and R. Sankaranarayanan, "Cancer of the cervix uteri," International Journal of Gynecology \& Obstetrics, vol. 143, pp. 22-36, 2018.

[8] F. Lécuru, P. Mathevet, D. Querleu et al., "Bilateral negative sentinel nodes accurately predict absence of lymph node metastasis in early cervical cancer: results of the SENTICOL study," Journal of Clinical Oncology, vol. 29, no. 13, pp. 1686-1691, 2011.

[9] B. Cormier, J. P. Diaz, K. Shih et al., "Establishing a sentinel lymph node mapping algorithm for the treatment of early cervical cancer," Gynecologic Oncology, vol. 122, no. 2, pp. 275-280, 2011.

[10] G. Salvo, P. T. Ramirez, C. F. Levenback et al., "Sensitivity and negative predictive value for sentinel lymph node biopsy in women with early-stage cervical cancer," Gynecologic Oncology, vol. 145, no. 1, pp. 96-101, 2017.

[11] R. M. Schols, N. J. Connell, and L. P. S. Stassen, "Near-infrared fluorescence imaging for real-time intraoperative anatomical guidance in minimally invasive surgery: a systematic review of the literature," World Journal of Surgery, vol. 39, no. 5, pp. 1069-1079, 2015.

[12] A. Buda, B. Bussi, G. Di Martino et al., "Sentinel lymph node mapping with near-infrared fluorescent imaging using indocyanine green: a new tool for laparoscopic platform in patients with endometrial and cervical cancer," Journal of Minimally Invasive Gynecology, vol. 23, no. 2, pp. 265-269, 2016.

[13] B. Hagen, M. Valla, G. Aune et al., "Indocyanine green fluorescence imaging of lymph nodes during robotic-assisted laparoscopic operation for endometrial cancer. A prospective validation study using a sentinel lymph node surgical algorithm," Gynecologic Oncology, vol. 143, no. 3, pp. 479-483, 2016.

[14] Y. Diab, "Sentinel lymph nodes mapping in cervical cancer a comprehensive review," International Journal of Gynecologic Cancer, vol. 27, no. 1, pp. 154-158, 2017.

[15] S. Imboden, A. Papadia, M. Nauwerk et al., "A comparison of radiocolloid and indocyanine green fluorescence imaging, sentinel lymph node mapping in patients with cervical cancer undergoing laparoscopic surgery," Annals of Surgical Oncology, vol. 22, no. 13, pp. 4198-4203, 2015.

[16] I. Ruscito, M. L. Gasparri, E. I. Braicu et al., "Sentinel node mapping in cervical and endometrial cancer: indocyanine green versus other conventional dyes-A meta-analysis," $A n$ nals of Surgical Oncology, vol. 23, no. 11, pp. 3749-3756, 2016.

[17] S. J. Seong, H. Park, K. M. Yang et al., "Detection of sentinel lymph nodes in patients with early stage cervical cancer," Journal of Korean Medical Science, vol. 22, no. 1, pp. 105-109, 2007.

[18] M. Bedyńska, G. Szewczyk, T. Klepacka et al., "Sentinel lymph node mapping using indocyanine green in patients with uterine and cervical neoplasms: restrictions of the method," Archives of Gynecology and Obstetrics, vol. 299, no. 5, pp. 1373-1384, 2019.
[19] S. Malur, N. Krause, C. Köhler, and A. Schneider, "Sentinel lymph node detection in patients with cervical cancer," Gynecologic Oncology, vol. 80, no. 2, pp. 254-257, 2001.

[20] J. A. P. Leijte, I. M. C. van der Ploeg, R. A. Valdés Olmos, O. E. Nieweg, and S. Horenblas, "Visualization of tumor blockage and rerouting of lymphatic drainage in penile cancer patients by use of SPECT/CT," Journal of Nuclear Medicine, vol. 50, no. 3, pp. 364-367, 2009.

[21] U. Veronesi, G. Paganelli, V. Galimberti et al., "Sentinel-node biopsy to avoid axillary dissection in breast cancer with clinically negative lymph-nodes," The Lancet, vol. 349, no. 9069, pp. 1864-1867, 1997.

[22] D. Krag, D. Weaver, T. Ashikaga et al., "The sentinel node in breast cancer - a multicenter validation study," New England Journal of Medicine, vol. 339, no. 14, pp. 941-946, 1998.

[23] C. Altgassen, H. Hertel, A. Brandstädt, C. Köhler, M. Dürst, and A. Schneider, "Multicenter validation study of the sentinel lymph node concept in cervical cancer: AGO Study Group," Journal of Clinical Oncology, vol. 26, no. 18, pp. 2943-2951, 2008.

[24] N. Bizzarri, L. Pedone Anchora, G. F. Zannoni et al., "Role of one-step nucleic acid amplification (OSNA) to detect sentinel lymph node low-volume metastasis in early-stage cervical cancer," International Journal of Gynecologic Cancer, vol. 30, no. 3, pp. 364-371, 2020. 